\title{
Prone position in wards for spontaneous breathing Covid-19 patients: a retrospective study
}

\author{
Johan Wormser $^{1}$ (D) $\cdot$ Christophe Romanet $^{1} \cdot$ François Philippart ${ }^{1,2}$ (D) \\ Received: 23 October 2020 / Accepted: 14 December 2020 / Published online: 15 January 2021 \\ (C) Royal Academy of Medicine in Ireland 2021
}

\begin{abstract}
The pandemic of coronavirus disease 2019 (Covid-19) caused a large number of non-ventilated hypoxemic patients to require the use of prone position. The aim of this study is to measure the efficiency and tolerance of prone positioning in ward hypoxemic patients treated for Covid-19. This retrospective study included confirmed Covid-19 hypoxemic patients treated by at least one prone position session. Primary outcome was pulse oximetry over inspired oxygen fraction ratio $\left(\mathrm{SpO}_{2} / \mathrm{FiO}_{2}\right)$ before, during, and after prone position. Secondary outcomes were failure, adverse events, and poor tolerance rate. Twenty-seven patients were included. During first, second and third sessions, $\mathrm{SpO}_{2} / \mathrm{FiO}_{2}$ ratio was significantly higher during posture than before $(p<$ $0.0001, p<0.01$, and $p<0.001$ respectively). Eighteen patients were responders (defined as an improvement of $\mathrm{SpO}_{2} / \mathrm{FiO}_{2}$ of more than 50) during the first posture and have a shorter length of hospital stay than non-responder patients. Failure rate was 5\%, and poor tolerance and adverse events rates were $8 \%$ and $7 \%$ respectively. Our study found that prone position in wards improved alveolar exchange during posture and is well tolerated. This technique could be used in any medical ward.
\end{abstract}

Keywords Acute respiratory failure $\cdot$ Covid- $19 \cdot$ Hypoxemia $\cdot$ Oxygenation $\cdot$ Tolerance $\cdot$ Wards

\section{Introduction}

The coronavirus disease 2019 (Covid-19) pandemia expansion was responsible for an overload of patients who developed an acute respiratory failure [1]. Many critical hypoxemic patients were treated in wards due to lack of intensive care units (ICU) bed availability. Despite the data scarcity in conscious nonmechanically ventilated patients, prone positioning was broadly suggested in this context. The aim of our study was to evaluate the efficiency and tolerance of prone positioning in non-ICU patients.

\section{Methods}

We conducted a retrospective study in Groupe Hospitalier Paris Saint-Joseph wards between March 15 and July 6, 2020.

Johan Wormser

jwormser@ghpsj.fr

1 Intensive Care Unit, Groupe Hospitalier Paris Saint-Joseph, Paris, France

2 Institute for Integrative Cell Biology, Microbiology: Endotoxins, Structures and Host Response, Gif-sur-Yvette, France
Confirmed Covid-19 hypoxemic patients who benefited from at least one prone position were included. Patients were not included if they had less than $4 \mathrm{~L} / \mathrm{min}$ oxygen flow. This study was approved by the Groupe Hospitalier Paris Saint-Joseph Institutional Review Board and registered following the French Reference Methodology MR-004. Anonymous data were collected in the absence of opposition of the patients to the use of their data.

The main outcome was the $\mathrm{SpO}_{2} / \mathrm{FiO}_{2}$ evolution, estimated by pulse oximetry $\left(\mathrm{SpO}_{2}\right)$ and inspired oxygen fraction $\left(\mathrm{FiO}_{2}\right)$ collected immediately before, during, and after each set up. We also compared patients who responded and those who did not: responders were defined as an improvement of $\mathrm{SpO}_{2} / \mathrm{FiO}_{2}$ of more than 50. Secondary outcomes were (i) immediate failure to sustain posture, (ii) adverse events (desaturation, modification in blood pressure or heart rate, vomiting) during prone position, and (iii) poor-tolerance (impossibility to withstand the position due to subjective reasons such as onset or increase of pain, worsening of dyspnea, uncomfort, or anxiety).

Main outcome analysis was made using Friedman paired tests and Dunn's post-test. Continuous variables were presented as median with interquartile range (IQR) due to their distribution. Categorical variables were expressed as numbers with percentages. Statistics were processed using the $\mathrm{R}$ 
software with a two-sided 5\% significance threshold. When necessary, $95 \%$ confidence intervals $(95 \% \mathrm{CI})$ are presented. Figures were created using GraphPad Prism 8 software.

\section{Results}

We included 27 patients out of 38 eligible patients: 11 patients were not included because flow oxygen was under $4 \mathrm{~L} / \mathrm{min}$ at the first prone position.

The median age of patients was 73 years (IQR, 60-79). Fifty-nine percent were male $(n=16)$ and the median body mass index (BMI) was 28.1 (IQR, 25.4-32.8). The most common comorbidities were arterial hypertension $(n=13,48 \%)$, chronic obstructive pulmonary disease $(n=7,26 \%)$, and type II diabetes $(n=6,22 \%)$. One patient $(4 \%)$ was an active smoker while 13 patients were former smokers $(48 \%)$. The remaining patients had never smoked $(n=13,48 \%)$.

Lesion severity on computerized tomography scan was moderate $(10-25 \%)$ for 7 patients $(26 \%)$, extensive (25$50 \%)$ for 10 patients $(37 \%)$, severe $(50-75 \%)$ for 8 patients $(30 \%)$, and critical (over $75 \%$ ) for one patient (4\%). The first prone position was achieved at a median duration of 2 days after hospital admission. Seventy-four percent of patients $(n=$ 20) were on oxygen flow rates of at least $6 \mathrm{~L} / \mathrm{min}$ and $\mathrm{SpO}_{2} /$ $\mathrm{FiO}_{2}$ median ratio was 187.5 (IQR, 161.6-211.2). Median duration of hospital length of stay was 16 days (IQR, 9-24). One patient died during hospitalization.

Twenty-four patients completed the first prone position: the median $\mathrm{SpO}_{2} / \mathrm{FiO}_{2}$ ratio was 342.5 (238.9-438.1) which was significantly higher than the $188.5(162.5-216.9)$ before prone position $(p<0.0001)$. There was no difference in $\mathrm{SpO}_{2} / \mathrm{FiO}_{2}$ before and after posturing: 188.5 (162.5-216.9)
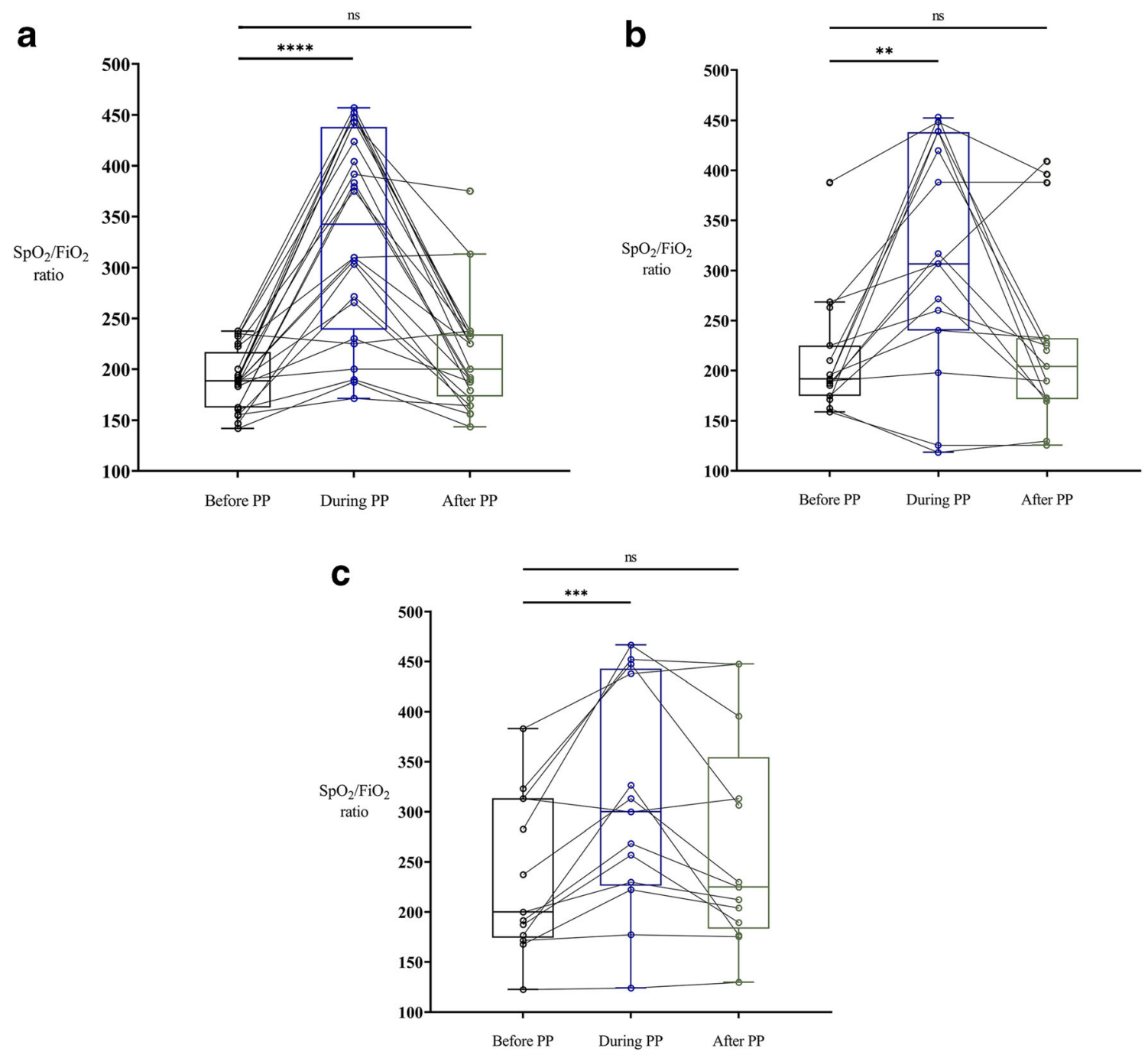

Fig. $1 \mathrm{SpO}_{2} / \mathrm{FiO}_{2}$ ratio before, during, and after prone position. a First prone position $(n=24)$ : $* * * * p<0.0001$ between before and during prone position. b Second prone position $(n=15)$ : ${ }^{*} p<0.01$ between before and during prone position. $\mathbf{c}$ Third prone position $(n=13)$ : ***p $p 0.001$ between before and during prone position. PP, prone position; ns, nonsignificance 
vs 200.0 (173.4-234.4). Similar results were found during the next posture sessions. Results are presented in Fig. 1.

During the first posture, 18 patients were responders. Moreover, responder and non-responder patients did not differ from one another, except for length of hospital stay, which is shorter for responders (Table 1).

Considering the 64 episodes of postures, no serious adverse event has occurred. Three postures were impossible to sustain immediately, 2 for anxiety, and 1 for desaturation: the failure rate of prone position was estimated to be $5 \%$ (95\% CI, 1$13 \%)$. Among the 61 sessions, 7 desaturations have occurred: the adverse event rate was estimated to be $7 \%$ (95\% CI, 2$16 \%)$. Finally, the prone position poor-tolerance frequency was estimated to be $8 \%$ (95\% CI, 3-18\%): 2 for onset of pain and 3 for severe uncomfort.

\section{Discussion}

Our results confirm that prone position improved Covid-19 patients' alveolar oxygen exchange in ward patients and is well tolerated.
Several studies have been recently published about prone position in Covid-19 patients. Elharrar et al. have yet ascertained that only $25 \%$ were responders to prone position [2]. However, our study showed more promising results: other studies have found the same trends as ours in oxygenation improvement during the posture [3-5]. Sartini et al. found that 12 of 15 patients had persistent oxygenation improvement after the posture's end [3]. In our study, oxygenation improvement did not seem to persist when the position was stopped; therefore, our results seem consistent with findings from previous studies $[2,4]$. There is some heterogeneity in literature regarding oxygenation improvement during and after posture: this could be due to a substantial difference in time measurement itself or because outcomes, populations (e.g., age and body mass index), and associated treatments (e.g., noninvasive ventilation during posture) were different.

The results of our secondary outcomes were consistent with previous findings and suggested that difficulties encountered are rather scarce and mild $[2-4,6]$.

There are several limitations in this study. Firstly, it was a retrospective study from a single center. Moreover, homogeneity of patients and unicity of the pathology made the results transposable in other locations. Secondarily, the $\mathrm{SpO}_{2} / \mathrm{FiO}_{2}$
Table 1 Characteristics of responder and non-responder patients

\begin{tabular}{|c|c|c|c|}
\hline Characteristics & $\begin{array}{l}\text { First PP responders } \\
(n=18)\end{array}$ & $\begin{array}{l}\text { First PP non-responders } \\
(n=6)\end{array}$ & $p$ value \\
\hline Age, median (IQR) & $71(61.2-80.0)$ & $64.5(46.5-76.5)$ & 0.404 \\
\hline \multicolumn{4}{|l|}{ Sex, No. (\%) } \\
\hline Female & $7(39)$ & $4(67)$ & \multirow[t]{2}{*}{0.357} \\
\hline Male & $11(61)$ & $2(33)$ & \\
\hline BMI, median (IQR) ${ }^{\mathrm{a}}$ & $28.1(25.4-32.0)$ & $25.6(24.1-25.9)$ & 0.173 \\
\hline \multicolumn{4}{|l|}{ Smoking history, No. (\%) } \\
\hline Active smoker & $1(6)$ & $0(0)$ & \multirow{3}{*}{0.745} \\
\hline Former smoker & $8(44)$ & $2(33)$ & \\
\hline Never smoked & $9(50)$ & $4(67)$ & \\
\hline \multicolumn{4}{|l|}{ Comorbidities, No. (\%) } \\
\hline Chronic obstructive pulmonary disease & $4(22)$ & $1(17)$ & 1 \\
\hline Hypertension & $9(50)$ & $1(17)$ & 0.341 \\
\hline Type II diabetes & $2(11)$ & $2(33)$ & 0.251 \\
\hline \multicolumn{4}{|l|}{ Severity of CT damage, No. $(\%)^{\mathrm{a}}$} \\
\hline Moderate $(10-25 \%)$ & $4(24)$ & $2(33)$ & \multirow[t]{4}{*}{0.426} \\
\hline Extensive $(25-50 \%)$ & $7(41)$ & $2(33)$ & \\
\hline Severe $(50-75 \%)$ & $6(35)$ & $1(17)$ & \\
\hline Critical $(>75 \%)$ & $0(0)$ & $1(17)$ & \\
\hline \multicolumn{4}{|l|}{ Medical treatment at inclusion, No. $(\%)$} \\
\hline Hydroxychloroquine & $9(50)$ & $4(67)$ & 0.649 \\
\hline Azithromycin & $13(72)$ & $4(67)$ & 1 \\
\hline Anakinra & $13(72)$ & $3(50)$ & 0.362 \\
\hline Antibiotics & $17(94)$ & $4(67)$ & 0.143 \\
\hline $\mathrm{SpO}_{2} / \mathrm{FiO}_{2}$ ratio before first $\mathrm{PP}$, median (IQR) & $190.6(185.9-216.9)$ & $172.0(156.7-188.0)$ & 0.182 \\
\hline Oxygen flow before first PP, median (IQR) & $6(4.5-6)$ & $7.5(6.0-9.0)$ & 0.321 \\
\hline$<6 \mathrm{~L} / \mathrm{min}$, No. $(\%)$ & $5(28)$ & $1(17)$ & 1 \\
\hline$\geq 6 \mathrm{~L} / \mathrm{min}$, No. $(\%)$ & $13(72)$ & $5(83)$ & \\
\hline Length of stay in hospital, median (IQR) & $10.50(8.25-18.25)$ & $26(19.00-35.25)$ & 0.013 \\
\hline
\end{tabular}

A responder to the posture was considered as such by an improvement of the measured $\mathrm{SpO}_{2} / \mathrm{FiO}_{2}$ ratio by at least 50 points. ${ }^{\mathrm{a}}$ Missing data: BMI for 3 patients and 1 for $\mathrm{CT}$ scan severity. IQR, interquartile range; $B M I$, body mass index; $P P$, prone position; $\mathrm{CT}$, computerized tomography; $\mathrm{SpO}_{2}$, pulse oximetry; $\mathrm{FiO}_{2}$, inspired oxygen fraction 
ratio probably lacks precision but is correlated with $\mathrm{PaO}_{2}$ / $\mathrm{FiO}_{2}$ ratio [7] and is quite less invasive for conscious patients. Finally, the absence of randomization reduced our level of evidence; nonetheless, the variations of $\mathrm{SpO}_{2} / \mathrm{FiO}_{2}$ during and after prone position tend to confirm the efficiency and the transient nature of the effect.

In conclusion, prone position is easy to implement in wards, improves alveolar exchange during posture, and is well tolerated. Results need confirmation in randomized and highquality studies. Moreover, the benefit of this technique on intubation or mortality is currently unknown.

Acknowledgments We would like to thank Marie Lavigne, Layla Ninot, Aurel Bellaïche and all the physiotherapists, nurses, and health-care staff involved in the implementation of prone position with Covid-19 patients.

Author contributions JW, CR, and FP conceived and planned the following research. JW performed the data collection, the statistics analysis, and designed the figure with support from FP. JW and CR wrote the manuscript with support from FP. All authors provided critical feedback of the research and manuscript.

Data availability The datasets used and/or analyzed during the current study are available from the corresponding author on reasonable request.

\section{Compliance with ethical standards}

Conflict of interest The authors declare that they have no conflict of interest.

Ethics approval This study was approved by the Paris Saint-Joseph Hospital Institutional Review Board (IRB 00012157) and registered following the French Reference Methodology MR-004.
Consent to participate Anonymous data were collected in the absence of opposition of the patients to the use of their data.

\section{References}

1. Ceylan Z (2020) Estimation of COVID-19 prevalence in Italy, Spain, and France. Sci Total Environ 729:138817. https://doi.org/10.1016/j. scitotenv.2020.138817

2. Elharrar X, Trigui Y, Dols AM et al (2020) Use of prone positioning in nonintubated patients with COVID-19 and hypoxemic acute respiratory failure. JAMA 323(22):2336-2338. https://doi.org/10. 1001/jama.2020.8255

3. Sartini C, Tresoldi M, Scarpellini P et al (2020) Respiratory parameters in patients with COVID-19 after using noninvasive ventilation in the prone position outside the intensive care unit. JAMA 323(22): 2338-2340. https://doi.org/10.1001/jama.2020.7861

4. Coppo A, Bellani G, Winterton D et al (2020) Feasibility and physiological effects of prone positioning in non-intubated patients with acute respiratory failure due to COVID-19 (PRON-COVID): a prospective cohort study. Lancet Respir Med 8(8):765-774. https://doi. org/10.1016/S2213-2600(20)30268-X

5. Caputo ND, Strayer RJ, Levitan R (2020) Early self-proning in awake, non-intubated patients in the emergency department: a single ED's experience during the COVID-19 pandemic. Acad Emerg Med 27(5):375-378. https://doi.org/10.1111/acem.13994

6. Ng Z, Tay WC, Ho CHB (2020) Awake prone positioning for nonintubated oxygen dependent COVID-19 pneumonia patients. Eur Respir J 56(1):2001198. https://doi.org/10.1183/13993003.011982020

7. Rice TW, Wheeler AP, Bernard GR et al (2007) Comparison of the $\mathrm{SpO} 2 / \mathrm{FIO} 2$ ratio and the $\mathrm{PaO} 2 / \mathrm{FIO} 2$ ratio in patients with acute lung injury or ARDS. Chest 132(2):410-417. https://doi.org/10.1378/ chest.07-0617

Publisher's note Springer Nature remains neutral with regard to jurisdictional claims in published maps and institutional affiliations. 